\title{
ANIRIDIA CONGÉNITA
}

\author{
Adriana Solano MD*, Juan F. DíazGranados MD**, Ángela Castillo MD***
}

\section{Resumen}

Objetivo: presentar un caso de aniridia congénita bilateral asociada con catarata e hipoplasia foveal y revisar la literatura existente. Métodos: revisión de la literatura y de la historia clínica de un paciente que consultó al Hospital de San José de Bogotá DC. Conclusiones: la aniridia es una alteración panocular, bilateral, muy poco frecuente que afecta la córnea, cámara anterior, iris, cristalino, retina, mácula y nervio óptico. Tiene una incidencia entre 1:64.000 y 1:100.000. Puede encontrarse de manera aislada o en asociación con otros síndromes, incluyendo el tumor de Wilms, anormalidades genitourinarias y retardo mental (síndrome de WARG).

Palabras clave: aniridia, nistagmus congénito, catarata congénita, hipoplasia foveal.

\section{CONGENITAL ANIRIDIA}

\begin{abstract}
Objective: to present one case of bilateral congenital aniridia associated with cataracts and foveal hypoplasia and conduct a review of available literature. Methods: a review of literature and clinical record of a patient who consulted Hospital de San José, Bogotá DC. Conclusions: aniridia is a rare panophthalmic disorder which involves the cornea, anterior chamber, iris, lens, retina, macula and optic nerve. Its incidence is between 1:64.000 and 1:100.000. It may occur alone or associated with other syndromes, such as Wilms tumor, genitourinary abnormalities and mental retardation (WARG Syndrome).
\end{abstract}

Key words: aniridia, congenital nystagmus, congenital cataract, foveal hypoplasia.

Fecha recibido: marzo 10 de 2011 - Fecha aceptado: junio II de 2011

* Médica oftalmóloga, subespecialista en oftalmología pediátrica y estrabismo. Hospital de San José. Instructor Asociado, Fundación Universitaria de Ciencias de la Salud, Bogotá DC, Colombia,
** Médico oftalmólogo, subespecialista en córnea y cirugía refactiva, Jefe del Servicio de Oftalmología, Hospital de San José. Profesor Asociado, Fundación Universitaria de Ciencias de la Salud, Bogotá DC, Colombia.

*** Residente I de Oftalmología. Fundación Universitaria de Ciencias de la Salud, Bogotá DC, Colombia. 


\section{Introducción}

Paciente de sexo masculino de 22 años con antecedente de retardo en el desarrollo psicomotor, que presenta cuadro de mala agudeza visual en ojo derecho desde hace 19 años. Al examen físico se encontró agudeza visual 20/800 en ambos ojos, nistagmus horizontal bilateral, conjuntivalización de la córnea periférica y aniridia bilateral, opacidad subcapsular posterior densa en ojo derecho, pseudofaquia en ojo izquierdo con opacidad de cápsula posterior e hipoplasia foveal en ambos ojos.

Generalidades: la aniridia es la ausencia de una gran porción del iris. El término no es exacto debido a que una parte de la raíz del iris está siempre presente. En la mayoría de los casos es bilateral y cerca del $90 \%$ se asocia con otras malformaciones oculares, por lo que se considera un defecto panocular. En el 10\% de los casos se encuentra aislada. Fue descrita por primera vez en 1818 por Barrata. Es una patología rara, con una incidencia entre 1:64.000 y 1:100.000 nacidos vivos. La morbilidad en la aniridia es con frecuencia elevada, por el compromiso de la visión y las alteraciones sistémicas asociadas en especial con el tumor de Wilms.

Genética: se puede presentar en tres formas como: 1) desorden autosómico, 2) deleción cromosómica identificable y 3 ) caso esporádico en cerca de la tercera parte de los casos. ${ }^{1-4}$ Cerca de dos tercios de las personas con aniridia tienen historia familiar, con herencia autosómica dominante y por lo regular no se asocian con el tumor de Wilms. ${ }^{5}$ Los genes implicados en la oculogénesis son varios, pero sin duda los más importantes son los PAX6, que codifican para un factor ranscripcional o proteína que interactúa con el DNA para regular la función de otros genes. El gen PAX6 está implicado en el desarrollo embrionario del ojo de numerosos animales, desde invertebrados como la mosca (Drosophila melanogaster) hasta los humanos. Se expresa también en el cerebro y el páncreas. ${ }^{1-4,6,7}$ Se sitúa en la región 11p13, donde se encuentra el gen AN2, responsable de la aniridia tipo II, que es la más frecuente. Contiguo se encuentran el gen WT1 responsable del tumor de Wilms y de las anomalías genitales, y otros implicados en el retraso psicomotor. La deleción de todos estos genes origina un síndrome de genes continuos conocido como WAGR $^{1-4,7,8}$ (Figura 1).

La alteración del gen PAX-6 también es responsable de otras malformaciones oculares como la anomalía de Peters, cataratas con distrofia corneana, hipoplasia foveal y ectopia pupilar.

Otros genes determinantes de la aniridia son el de la fosfatasa ácida que se encuentra en el brazo corto de cromosoma 2p distal (gen AN1- aniridia tipo I) y los genes PITX1 y PITX2. ${ }^{4}$ Se debe realizar estudio genético en casos de aniridia congénita aislada, sindrómica (síndrome de WAGR) o ante la presencia de cualquiera de los posibles fenotipos asociados a mutaciones en PAX6:

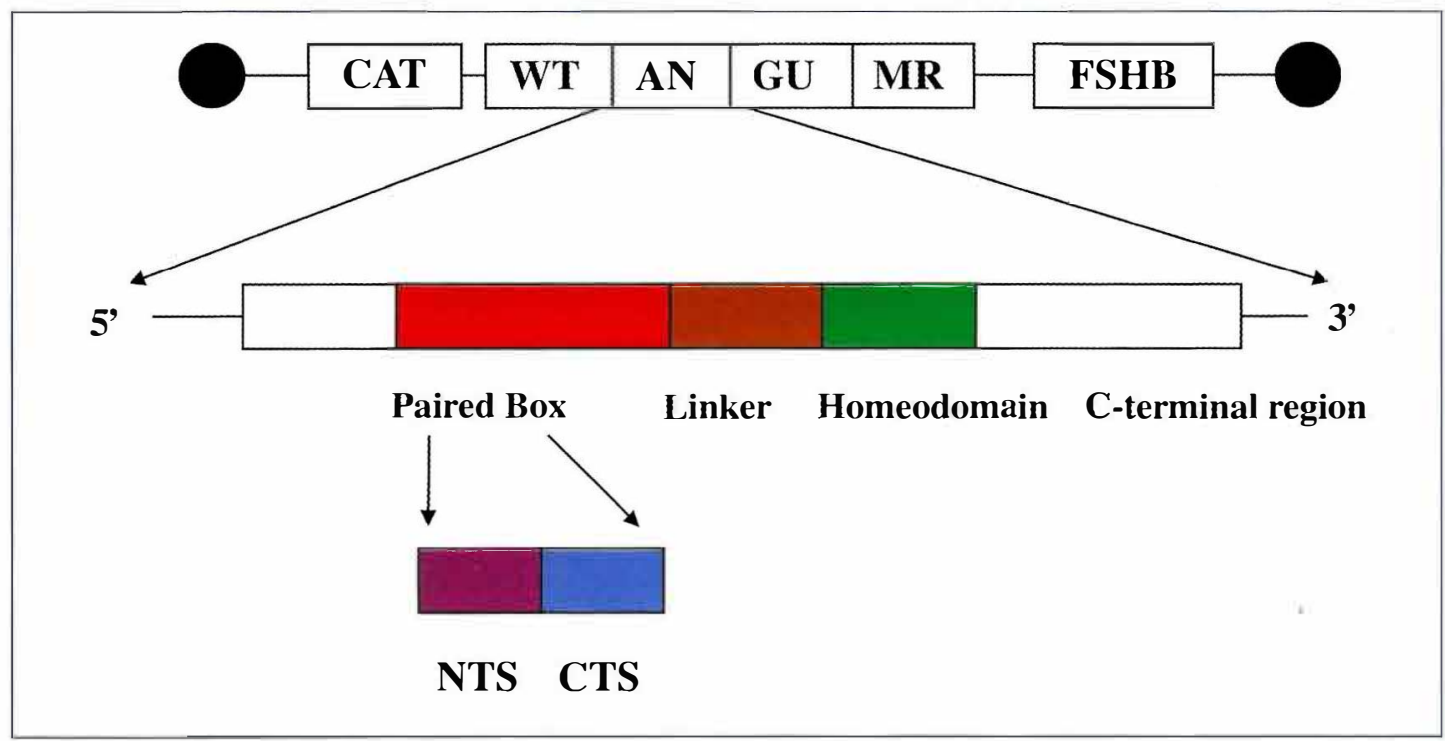

Figura I. Sindrome de genes continuos. 
anomalía de Peters, ectopia pupillae, hipoplasia foveal aislada o asociada con cataratas preseniles, queratopatía autosómica dominante relacionada con aniridia. Otras indicaciones menos frecuentes (y con resultado mutacional más improbable) son los colobomas (ocular o delnervio óptico) y la hipoplasia bilateral del nervio óptico. El estudio genético es imprescindible para conocer el riesgo de tumor de Wilms en los casos de aniridia congénita "de novo" y para realizar diagnóstico prenatal. ${ }^{7}$

\section{Compromiso ocular}

Agudeza visual: en general suele ser baja (niveles inferiores a 20/100,20/200). Es secundaria debido a la hipoplasia foveal o del nervio óptico. La visión también se afecta por otras manifestaciones del síndrome como la queratopatía, la catarata congénita o glaucoma. ${ }^{1-3.5}$

Motilidad ocular y visión binocular: la mayoría de los niñoscon aniridia tienen nistagmus, estrabismo, ambliopía o alteraciones de la fusión. ${ }^{1-3.59,9,10}$ El estrabismo es común, a menudo secundario a pérdida visual asimétrica o a anisometropía. $^{5}$

Córnea: la queratopatía asociada a aniridia es secundaria a una deficiencia de células stem y tiene una incidencia del 20\% ${ }^{3.7}$ Los signos aparecen en la primera década de la vida con engrosamiento y vascularización de la córnea periférica que en forma gradual avanza hacia la región central hasta comprometer toda la superficie, resultando en erosiones recurrentes, ulceración y fibrosis subepitelial con descompensación de la córnea y opacificación ${ }^{1,3.11}$ (Figura 2). Por lo regular empeora después de cirugías que impliquen manipulación excesiva del limbo o de la aplicación de antimetabolitos para el tratamiento del glaucoma. El manejo de la queratopatía asociada con aniridia va desde lágrimas artificiales hasta trasplante de limbo y/o de membrana amniótica de acuerdo con el estadio de la misma. ${ }^{3.7}$

El grosor del estroma corneano en los pacientes con aniridia congénita es mayor que el de la población normal. Brandt, Whitson y colaboradores describieron el espesor corneano central entre 630 y 690 micras en los pacientes con aniridia congénita, frente a 550 micras de media en un grupo control de población normal. ${ }^{12.13}$ Este dato debe

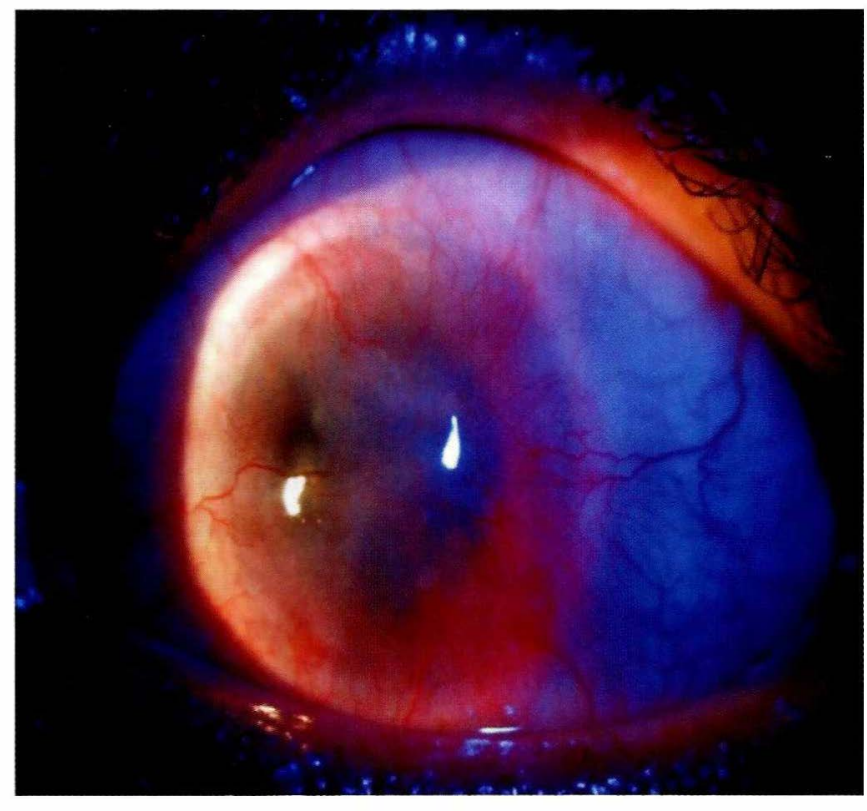

Figura 2. Deficiencia de limbo.

tenerse en cuenta al realizar mediciones de la presión intraocular. La microcórnea también puede asociarse con aniridia. $^{14,15}$

Glaucoma: la incidencia de glaucoma en la infancia de los pacientes con aniridia varía entre el $6 \%$ y $75 \%$ aumentando con la edad. Se produce debido a una migración progresiva de la raíz del iris hacia el ángulo iridocorneal, creando una obstrucción del trabéculo por la formación de sinequias. La severidad del glaucoma guarda relación con el grado de malformación del ángulo. Es muy raro que aparezca en la primera década de la vida, es en la segunda cuando surge la enfermedad ya que la migración anterior del iris es progresiva. En el recién nacido el ángulo está abierto, pero ya se pueden visualizar finos procesos y adherencias entre el estroma del iris y la malla trabecular (como si fueran sinequias), dando un aspecto "en dientes de sierra" entre la periferia del estroma del rudimento de iris, el espolón escleral y la banda ciliar de la malla trabecular. A lo largo de los años estas "adherencias" aumentan su consistencia, grosor y pigmentación y originan una migración anterior de la parte periférica del rudimento de iris cerrando el ángulo antes visible.

Existen también casos, aunque escasos, de pacientes con aniridia y ángulos abiertos que desarrollan glaucoma en la edad adulta. ${ }^{1.3 .11,16}$ Una vez se desarrolla el glaucoma 
en los pacientes con aniridia con frecuencia es refractario al tratamiento médico y quirúrgico. La trabeculectomía, la trabeculotomía, la goniotomía y la trabeculoplastia con laser argón ejercen poco control en la presión intraocular del glaucoma por aniridia (Figura 3). El tratamiento con procedimientos ciclodestructivos y los implantes de Molteno, están asociados con altas tasas de complicaciones y poreso no son una buena opción como terapia inicial. La goniocirugía profiláctica en pacientes con cambios tempranos a nivel angular es efectiva en prevenir o retardar la presentación del glaucoma. Swanner, Walton y Chen afirman que sin goniocirugía profiláctica se puede esperar glaucoma en la mitad de los pacientes con aniridia y es difícil de controlar. ${ }^{17}$

Cristalino, alteraciones de la posición: la subluxación del cristalino (ectopia lentis) es también frecuente en la aniridia. Se ha estimado que ocurre en al menos la mitad de los ojos y casi siempre consiste en un desplazamiento hacia arriba. En 1947 Beattie ${ }^{18}$ describió la subluxación superior en 12 casos de una familia de 28 miembros con aniridia, así como un riesgo mucho mayor de glaucoma en los casos con subluxación del cristalino. ${ }^{7,19}$ Es posible que esta última favorezca la formación de adherencias en elángulopor los movimientos delcristalino.Asímismo, la debilidad zonular podría estar relacionada con anomalías más severas del ángulo. La degeneración de la zónula en la aniridia es posible que se deba a la alteración (hipoplasia) del cuerpo ciliar, aspecto que ha sido valorado en la clínica mediante biomicroscopía

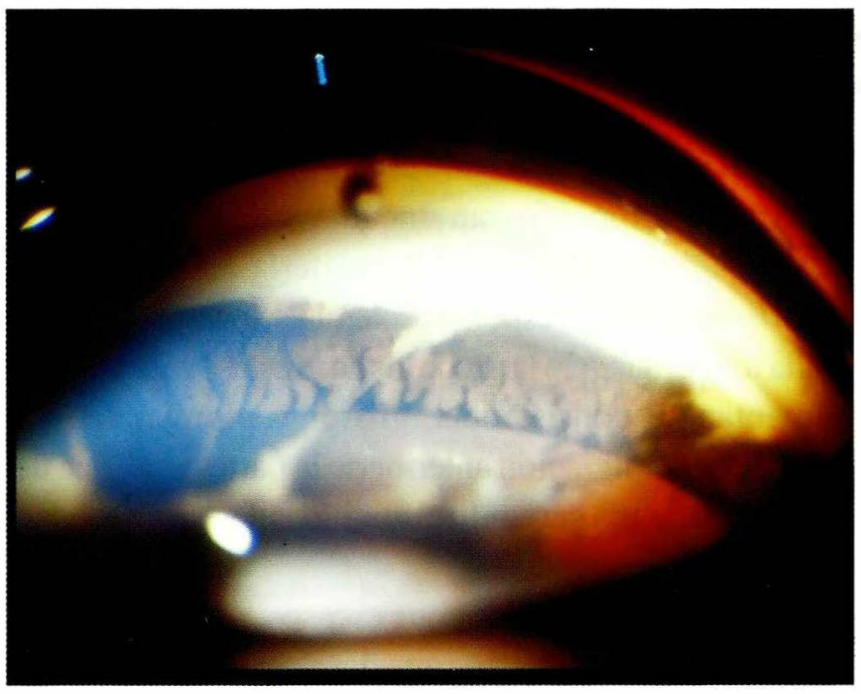

Figura 3. Remanente de iris en gonioscopia. ultrasónica, en donde se aprecia que las opacidades corticales en los cristalinos subluxados tienden a desarrollarse más en el sector con mayor defecto zonular. ${ }^{20}$ Otra alteración del cristalino es la presencia de adhesión queratolenticular, la cual se ha descrito asociada con aniridia y catarata. Es posible que se trate de un cuadro de disgenesia del segmento anterior similar a la anomalía de Peters, la cual también se ha relacionado en algunos casos con el gen $\operatorname{Pax6}^{21}$ (Figura 4).

Catarata: La aniridia se asocia con cataratas en un 50\% a $85 \%$ de los pacientes. Tienen morfología variable y se describen como polares, corticales, subcapsulares, lamelares y más raro nucleares. Es típico que existan desde el nacimiento pequeñas opacidades, las cuales no suelen causar un serio compromiso de la visión. En la mayoría de los casos, las opacidades progresan durante las dos primeras décadas de la vida hasta transformarse en cataratas subcapsulares, lamelares o corticales ${ }^{7}$ (Figura 5). Cuando las cataratas congénitas comprometen la visión, se recomienda su extracción antes de los res meses para evitar el nistagmus por deprivación sensorial. Sin embargo, no es fácil determinar el pronóstico visual debido a las demás alteraciones oculares asociadas. ${ }^{7}$ Estudios histológicos realizados enla cápsula anterior de pacientes con catarata y aniridia han encontrado que ésta es muy frágil; ${ }^{3.22 .23}$ la posible finura o fragilidad capsular correspondería a disminución del

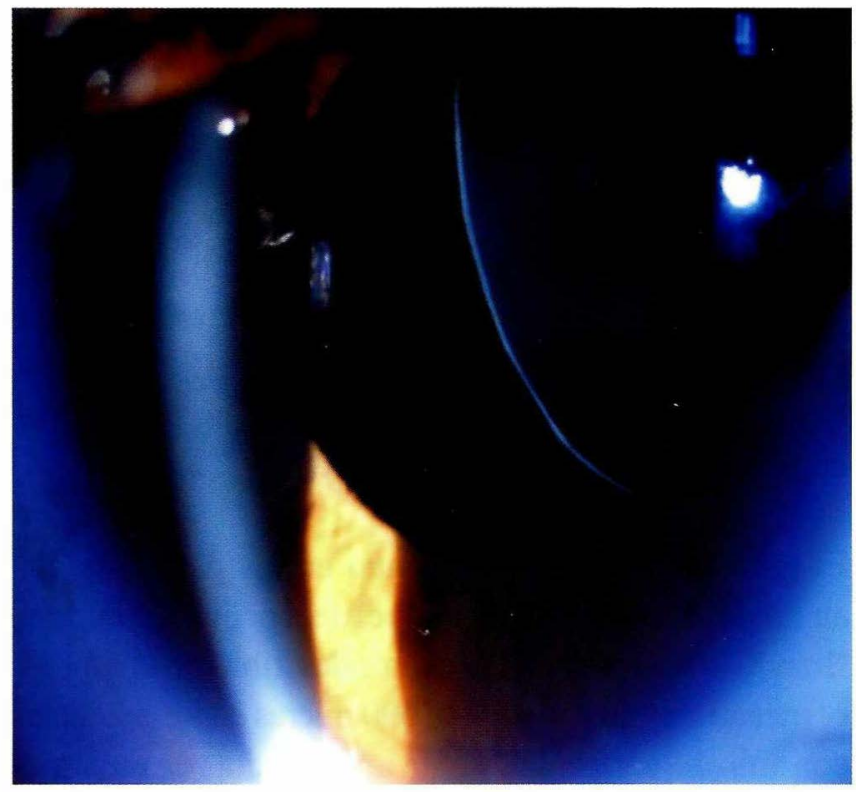

Figura 4. Luxación del cristalino. 
colágeno tipo IV, laminina, entactina, proteoglicano de heparansulfato y fibronectina. La cirugía de catarata con aniridia es complicada debido a las alteraciones patológicas asociadas como opacidades corneanas y cápsula frágil. ${ }^{3}$ Además se debe realizar reconstrucción de iris y pupila. ${ }^{22}$ También se han descrito complicaciones asociadas con el implante de lentes intraoculares en el sulcus ciliar. Se ha observado en estos ojos una alteración crónica de la barrera hematoacuosa (BHA) y se han postulado varias hipótesis para explicar dicha alteración: ${ }^{7}$

- el contacto de las hápticas y el diafragma del lente con los remanentes uveales puede producir irritación crónica

- el implante accidental de las hápticas del lente en el ángulo; un apoyo inadecuado en el ángulo iridocorneal favorecerá una mayor disfunción de la trabecular ya alterada, por ello se recomienda la práctica habitual de gonioscopia intraoperatoria para confirmar el correcto posicionamiento de las hápticas

- mayor movilidad del lente si se fija en el sulcus

- la barrera hematoacuosa en los ojos anirídicos podría ser más vulnerable a cualquier traumatismo en comparación con un ojo normal, la alteración crónica de esta barrera repercute sobre la presión intraocular y aunque el glaucoma es un problema intrínseco a la aniridia congénita, la alteración de la BHA puede favorecer su aparición o acelerar su progresión

Otras complicaciones asociadas con la alteración crónica de la BHA son inflamación crónica que puede condicionar la necesidad de emplear corticoides tópicos durante meses, pérdida progresiva de células endoteliales y edema macular microquístico. Por último, también se puede presentar el síndrome de fibrosis anterior progresiva. ${ }^{7,24}$ Los hallazgos iniciales incluyen la aparición de finas membranas que cubren las superficies anterior y posterior del lente intraocular (LIO), con posterior fibrosis y contracción de la cápsula provocando el desplazamiento anterior del LIO, todo esto en ausencia de inflamación observable en la clínica. Hallazgos tardíos incluyen el desarrollo progresivo de fibrosis con extensión anterior de la membrana, originando un mayor desplazamiento e inclinación del lente hacia la córnea y el cuerpo ciliar, causando hipotonía y desprendimientos traccionales de la retina. Un posible mecanismo para el desarrollo del síndrome de fibrosis anterior anirídico es el contacto o la proximidad de los lentes intraoculares con los vasos inmaduros del iris rudimentario de los ojos con aniridia congénita. Es aconsejable un seguimiento estrecho de aquellos pacientes con lentes intraoculares o a los que se les han realizado múltiples cirugías. El tratamiento incluye membranectomía precoz con el fin de prevenir las complicaciones asociadas con la extensión anterior y posterior de dicha fibrosis. ${ }^{7}$

Iris: la ausencia de iris está relacionada con disminución de la agudeza visual y fotofobia. Hay dos teorías de por qué no se desarrolla en forma apropiada; la ectodérmica, sugiere que la cúpula óptica no se desarrolla por completo y la mesodérmica hace relación a una alteración en la migración o proliferación de los elementos mesodérmicos ${ }^{2-6,9,10}$ (Figura 6). Se han ideado varias técnicas para el manejo de los síntomas secundarios a aniridia. Estas incluyen la cirugía de párpados, el uso de lentes de contacto de colores, tatuaje corneano y el implante de iris artificial. La más reciente es el uso de lente intraocular con diafragma de iris negro, para corregir la aniridia, congénita y traumática. ${ }^{25} \mathrm{El}$ implante de iris artificial fue introducido en Europa en 1994 por Reinhard y col. ${ }^{26}$ usando un lente intraocular con diafragma iridiano negro para el tratamiento de la aniridia congénita. Después Rosenthal presentó el primer implante de iris artificial a través de una pequeña incisión en córnea para el tratamiento de la disgenesia iridiana ${ }^{7}$ (Figura 7). Si bien es sabido que varios factores influyen en la baja visión de los pacientes anirídicos, la fotofobia es un síntoma

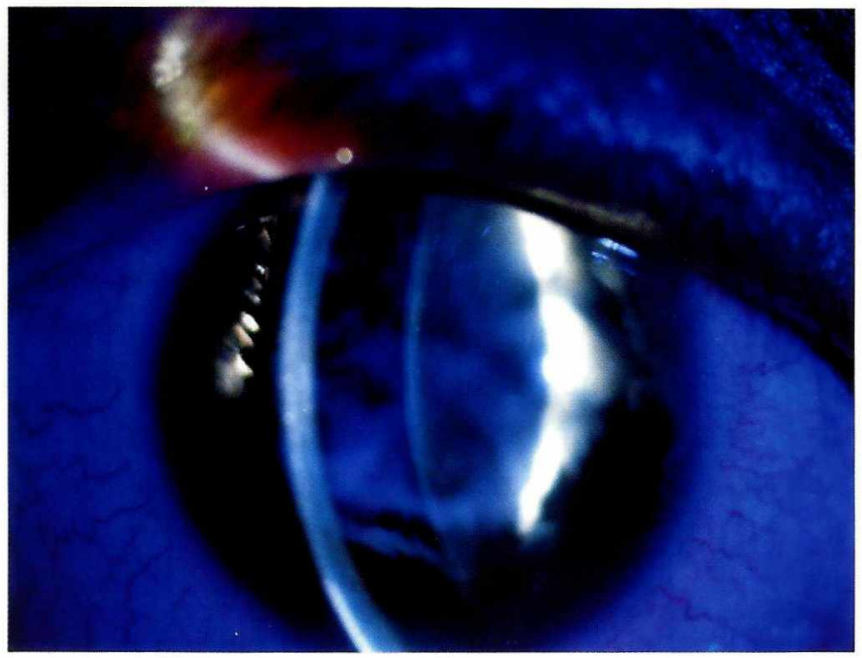

Figura 5. Catarata congénita y aniridia. 


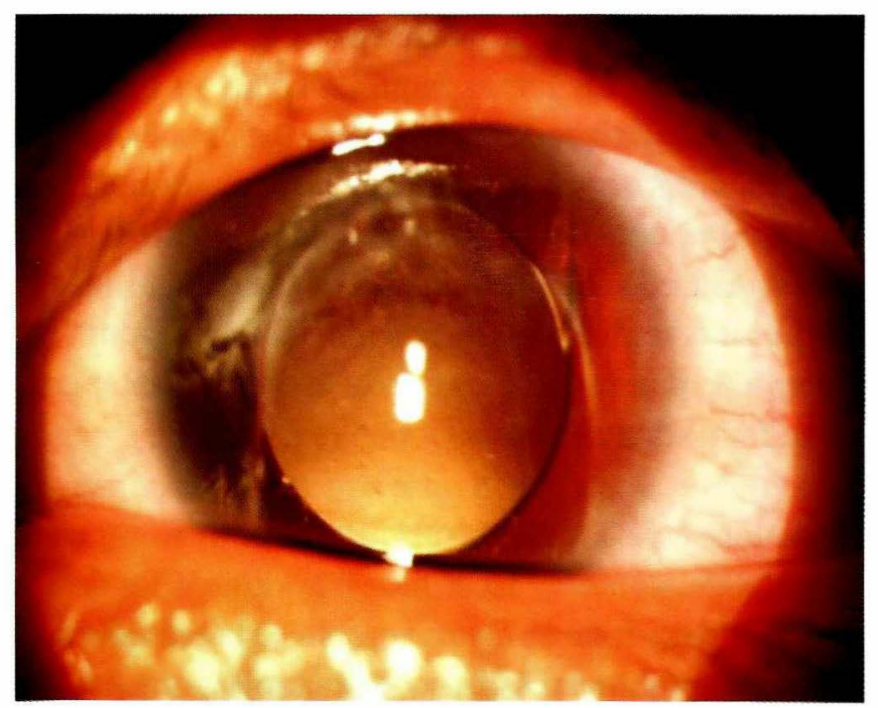

Figura 6. Lente intraocular y aniridia.

importante. La principal ventaja de poder insertar junto con el lente intraocular un dispositivo que actúe como iris artificial es que se va a reducir la cantidad de luz que entra en el ojo, con disminución de la fotofobia, mejoría en la profundidad de foco y menos aberraciones esféricas y cromáticas relacionadas con el borde del lente. ${ }^{27}$

Retina y nervio óptico: el $10 \%$ de los pacientes presenta grado variable de hipoplasia del nervio óptico que puede cursar sola o asociada con hipoplasia foveal (50\% de los casos). Se asocia con nistagmus pendular horizontal en la mayoría de los pacientes. ${ }^{5,10}$ La hipoplasia del nervio óptico es una anomalía congénita no progresiva, segmentaria o difusa, por lo regular bilateral. Se caracteriza por disminución del número de axones y células ganglionares de la retina con integridad de los elementos mesodérmicos de soporte glial del nervioóptico. En cortes histopatológicos, las células ganglionares y las fibras del nervio óptico están disminuidas en número, pero las capas externas de la retina son normales. ${ }^{28,29} \mathrm{~A}$ la oftalmoscopia se observa una papila pequeña (mitad o tercio del tamañonormal) y de coloración grisácea pálida. Es característico, aunque inconstante, el signo del doble anillo, consistente en un halo blanco-amarillento de hipopigmentación peripapilar causado por atrofia coriorretiniana concéntrica, rodeado a su vezdeotro anillo de hiperpigmentación. A pesar del pequeño tamaño del disco, los vasos sanguíneos retinianos suelen tener un calibre normal, aunque pueden ser tortuosos..$^{57,9,28-30} \mathrm{La}$ hipoplasia foveal es un signo cardinal en la aniridia y se caracteriza por una disminución o ausencia del reflejo foveal, una persistencia anormal de vasos en la zona avascular central y una cierta disminución de la pigmentación macular, observándose que la iluminación con luz azul no muestra el oscurecimiento central del pigmento de la mácula lútea. ${ }^{29}$ Estas anomalías foveales existen desde el nacimiento y en ocasiones permiten establecer el diagnóstico de aniridia en los casos leves. ${ }^{2}$ No parece existir relación entre la cantidad de iris e hipoplasia foveal. La aniridia también se asocia con desgarros retinianos y desprendimiento de retina., ${ }^{3,931,32}$

Síndromes asociados: la aniridia puede asociarse con enfermedades sistémicas; hasta $30 \%$ con tumor de Wilms, $5 \%$ con anomalías genitales y en $7 \%$ puede verse retraso mental. ${ }^{10}$ Dentro de los síndromes asociados se encuentran:

- El WAGR/WAGRO o de genes contiguos mencionado antes.

- El de Gillespie que cursa con aniridia parcial, ataxia cerebelosa y retraso mental. ${ }^{3,6.9}$

- El de Rieger que presenta disgenesia de iris y/o cámara anterior asociado con anomalías dentarias, malformaciones óseas, cardíacas, genitourinarias y del sistema nervioso central. El defecto de este

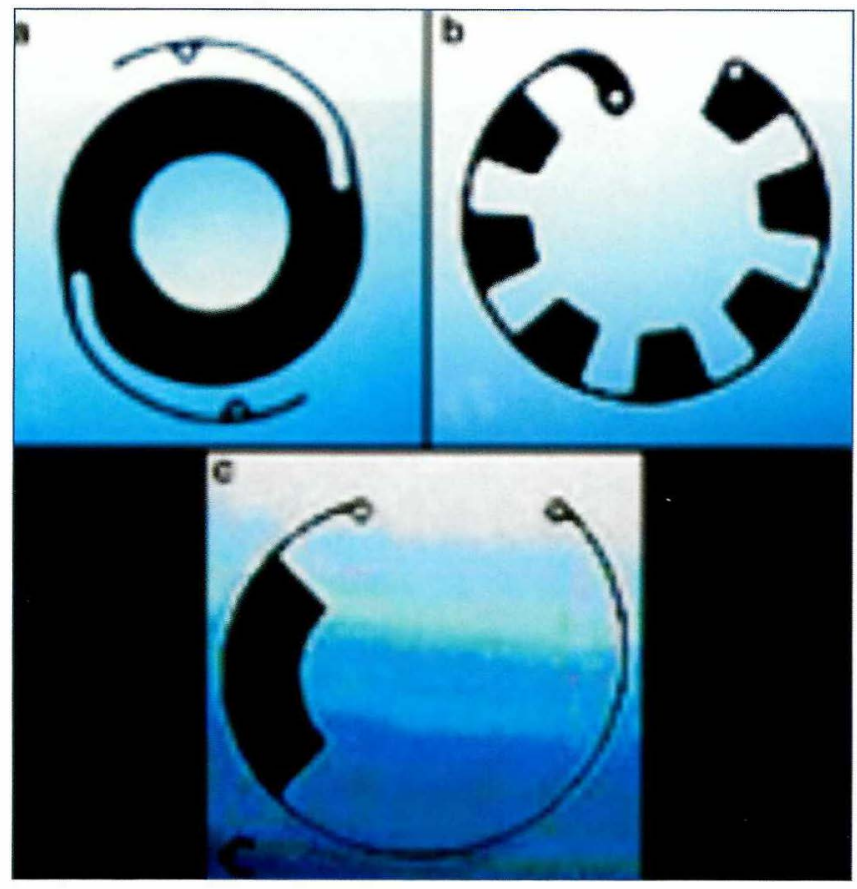

Figura 7. Lente intraocular con diafragma negro. 
síndrome se encuentra en el gen PITXl brazo largo del cromosoma 4 (4q25q26) y también hay otro gen PITX2 en el cromosoma 13. 2.4

Se ha descrito también una familia con agenesia e hipoplasia de rótula. ${ }^{4,10} \mathrm{La}$ aniridia puede verse sin ser un signo determinante en otros síndromes comola trisomía 13-síndrome de Patau, que presenta malformaciones cerebrales, faciales, cardíacas y renales que conducen rápido a la muerte. También en la trisomía parcial del cromosoma 12 y otras situaciones como el síndrome cerebro-ocular-genital, la aciduria láctica, el síndrome de Chadaverian-Kaplan y patrones malformativos únicos. ${ }^{2.3 .5 .6 .10}$

\section{Conclusiones}

Nuestro caso es un paciente con aniridia congénita asociada con alteraciones en la córnea, cristalino y retina. A pesar de la alta incidencia reportada en la literatura, no se encontró glaucoma. La única afección sistémica que se halló fue el retraso en el desarrollo psicomotor. Debido a que la aniridia es una patología que afecta el globo ocular de manera total, es muy importante realizar un examen detallado, oportuno y continuo para preservarla función visual y llevar a cabo un manejo interdisciplinario por encontrarse asociada con múltiples síndromes.

\section{Referencias}

1. Brauner SC, Walton DS, Chen TC. Aniridia. Int Ophthalmol Clin 2008; 48(2):7985

2. Ivanov I, Shuper A, Shohat M, Snir M, Weitz R. Aniridia: recent achievements in paediatric practice. Eur J Pediatr 1995; 154(10):795-800.

3. Lee H, Khan R, O' Keefe M. Aniridia: current pathology and management. Acta Ophthalmol 2008; 86(7):708-715.

4. Churchill A, Booth A. Genetics of aniridia and anterior segment dysgenesis. Br J Ophthalmol 1996; 80(7):669-673.

5. Tasman William JE. Congenital malformations of the eye. En: Duane's clinical ophthalmology on CD-ROM. 1999. Chapter 40.

6. Davis LK, Meyer KJ. Rudd DS, LibrantAL, Epping EA, Sheffield VC et al. Pax6 3' deletion results in aniridia, autism and mental retardation. Hum Genet 2008: 123(4):371-378.

7. Álvarez J, Gris O, Pérez J, Protocolo de actuación en pacientes con aniridia. Asociación española de aniridia. 2009. p. 1-213.

8. Fischbach BV, Trout KL, Lewis J, Luis CA, Sika M. WAGR syndrome: a clinical review of 54 cases. Pediatrics 2005; 116(4):984-988.

9. The eye M.D Association. Aniridia. En: Pediatric ophthalmology and strabismus. American Academy of Ophthalmology. 2003-2004.Chapter XX.

10. Asociación Española de Aniridia. Exposición aniridia. Arch. Soc. Esp. Oftalmol. 2009. Disponible en: http://www.aniridia.es.
11. Lopez-Garcia JS, Garcia-Lozano I, Rivas L, Martinez-Garchitorena J. [Congenital aniridia keratopathy treatment]. Arch Soc Esp Oftalmol 2006; 81(8):435-444.

12. Brandt JD, Casuso LA, Budenz DL. Markedly increased central comeal thickness: an unrecognized finding in congenital aniridia. Am J Ophthalmol 2004; 137: 348-350.

13. Whitson JT, Liang C, Godfrey DG, et al. Central corneal thickness in patients with congenital aniridia. Eye Contact Lens 2005; 31: 221-224.

14. Sugar J. Congenital stem cell deficiency. En: Holland EJ,Mannis MJ, eds, Ocular Surface Disease:Medical and Surgical Management. New York, Springer-Verlag, 2002; 93-99.

15. Beattie $\mathrm{PH}$ :.A consideration of aniridia, with a pedigree. $\mathrm{Br} \mathbf{J}$ Ophthalmol $1947 ; 31: 649$

16. Moreker M, Parikh R, Parikh SR, Thomas R. Aniridia associated with congenital aphakia and secondary glaucoma. Indian J Ophthalmol 2009; 57(4):313-314.

17. Swanner JC, Walton DS, Chen TC. Prevention of aniridic glaucoma with goniosurgery. Int Ophthalmol Clin 2004; 44(1):67-71.

18. Beauchamp GR. Anterior segment dysgenes keratolenticular adhesion and aniridia. J Pediat Ophthalmol Strabismus 1978; 17:55-8.

19. David R, MacBeath L, Jenkins T. Aniridia associated with microcomea and subluxated lenses. Br J Ophthalmol 1978; 62:118-121.

20. Hou ZQ, Hao YS, Wang W, et al. Clinical pathological study of the anterior lens capsule abnormalities in familial congenital aniridia with catarct. Beijing Da Xue Xue Bao 2005;37:494-497;Chinese.

21. Neuhann IM, Neuhann TF. Cataract surgery and aniridia. Curr Opin Ophthalmol 2010;21(1):60-64.

22. Tsai JH, Freeman JM, Chan C, Schwartz GS, Derby EA, Petersen MR, Holland EJ. A progressive anterior fibrus syndrome in patients with postsurgical congenital aniridia. Am J Ophtalmol 2005; 140 (6): 1075-1079.

23. Sundmacher R, Reinhard T, Althaus C. Black diaphragm intraocular lens in congenital aniridia. Ger J Ophtalmol 1994; 3:197-201.

24. Reinhard T, Engelhardt S, Sundmacher R. Black diaphragmaniridiaintraocular lens for congenital aniridia: long-term follow-up. J Cataract Refract Surg 2000;26(3):375-381.

25. Burk SE, Da Mata AP, Snyder ME, Cionni RJ, Cohen JS, Osher RH. Prosthetic iris implantation for confenital, traumatic, or functional iris deficiencies. J Cataract Refract Surg 2001; 27:1732-1740

26. Traboulsi EI. Optic nerve malformations. En: Huang D, Kaiser PK, Lowder CY, Traboulsi EI. Retinal imaging. Philadelphia, Elsevier Mosby, 2006; 591-593.

27. Kline LB. Optic nerve disorders. San Francisco, American Academy of Ophthalmology, 1996.

28. Kanski JJ, Damato B, Tanner V, Milewski SA. Diseases of the ocular fundus. Edinburgh, Elsevier Mosby, 2005.

29. Dowler JG, Lyons CJ \& Cooling RJ (1995): Retinal detachment and giant retinal tears in aniridia. Eye 9: 268-270.

30. Tagle RC. Congenital, developmental, and degenerative disorders of the iris and ciliary body. En: Albert DM, Jakobiec FA. Principles and practice of ophthalmology: basic sciences. Philadelphia,W.B. Saunders Company, 2000;1151-1175.

31. Okamoto F, Nakano S, Okamoto C, Hommura S, Oshika T. Ultrasound biomicroscopic findings in aniridia. Am J Ophthalmol 2004; 137:858-62.

32. McGuire DE, Weinreb RN, Goldbaum MH. Foveal hypoplasia demonstrated in vivo with optical coherence tomography. Am J Ophthalmol 2003; 135 (1): $112-4$. 